Journal for ImmunoTherapy of Cancer

\section{sequencing for unbiased tumor mutational burden estimation in samples with low tumor purity}

To cite: Hong TH, Cha $\mathrm{H}$, Shim JH, et al. Clinical advantage of targeted sequencing for unbiased tumor mutational burden estimation in samples with low tumor purity. Journal for ImmunoTherapy of Cancer 2020;8:e001199. doi:10.1136/jitc-2020-001199

- Additional material is published online only. To view please visit the journal online (http://dx.doi.org/10.1136/jitc2020-001199).

THH, HC and JHS contributed equally.

Accepted 21 September 2020

Check for updates

(c) Author(s) (or their employer(s)) 2020. Re-use permitted under CC BY. Published by BMJ.

For numbered affiliations see end of article.

Correspondence to Dr Woong-Yang Park; woongyang@gmail.com

Dr Se-Hoon Lee; sehoon.lee119@gmail.com

\section{ABSTRACT}

Background Tumor mutational burden (TMB) measurement is limited by low tumor purity of samples, which can influence prediction of the immunotherapy response, particularly when using whole-exome sequencing-based TMB (WTMB). This issue could be overcome by targeted panel sequencing-based TMB (pTMB) with higher depth of coverage, which remains unexplored.

Methods We comprehensively reanalyzed four public datasets of immune checkpoint inhibitor (ICl)-treated cohorts (adopting pTMB or wTMB) to test each biomarker's predictive ability for low purity samples (cut-off: 30\%). For validation, paired genomic profiling with the same tumor specimens was performed to directly compare wTMB and pTMB in patients with breast cancer (paired-BRCA, $n=165$ ) and ICl-treated patients with advanced non-small-cell lung cancer (paired-NSCLC, $\mathrm{n}=156$ ).

Results Low tumor purity was common (range 30\%$45 \%$ ) in real-world samples from ICl-treated patients. In the survival analyzes of public cohorts, wTMB could not predict the clinical benefit of immunotherapy when tumor purity was low (log-rank $p=0.874)$, whereas $p T M B$ could effectively stratify the survival outcome (log-rank $p=0.020$ ). In the paired-BRCA and paired-NSCLC cohorts, pTMB was less affected by tumor purity, with significantly more somatic variants identified at low allele frequency $(p<0.001)$. We found that wTMB was significantly underestimated in low purity samples with a large proportion of clonal variants undetected by whole-exome sequencing. Interestingly, pTMB more accurately predicted progression-free survival (PFS) after immunotherapy than wTMB owing to its superior performance in the low tumor purity subgroup ( $p=0.054$ vs $p=0.358$ ). Multivariate analysis revealed $\mathrm{pTMB}(\mathrm{p}=0.016)$, but not wTMB $(p=0.32)$, as an independent predictor of PFS even in lowpurity samples. The net reclassification index using pTMB was $21.7 \%$ in the low-purity subgroup $(p=0.016)$. Conclusions Our data suggest that TMB characterization with targeted deep sequencing might have potential strength in predicting $\mathrm{ICl}$ responsiveness due to its enhanced sensitivity for hard-to-detect variants at low- allele fraction. Therefore, pTMB could act as an invaluable biomarker in the setting of both clinical trials and practice outside of trials based on its reliable performance in mitigating the purity-related bias.

\section{BACKGROUND}

Immune checkpoint inhibitors (ICIs) have ushered in a new era of clinical oncology. The tumor mutational burden (TMB), devised as a surrogate for the neoantigen load, is increasingly being accepted as a relevant biomarker for predicting the response to ICI therapy. ${ }^{1-3}$ Nevertheless, the lack of harmonization across various next-generation sequencing (NGS) platforms and the limited predictive performance of tissue-based TMB estimates remain major barriers for precision immunogenetic approaches. ${ }^{4-6}$

Real-world clinical samples are inevitably and frequently associated with a limitation of low tumor content (ie, low purity). ${ }^{7}$ Anagnostou et al recently revealed the confounding impact of tumor purity on TMB estimates, emphasizing the need for an integrated biomarker that exhibits improved correlation with outcomes. ${ }^{8}$ This purity-related bias reduces TMB estimates in samples with low tumor purity, thereby increasing the risk of false-negative prediction for the response to ICIs. To effectively predict the response to ICIs on the basis of TMB, there is an urgent need to identify the causes and clinical consequences of the bias due to tumor purity.

Although considered the gold standard, whole-exome sequencing (WES)-based TMB (wTMB) estimation is not currently feasible or expedient in clinical settings. ${ }^{9}$ In theory, the 'gold standard' should be an accurate measurement of the total mutations that 
are capable of being recognized by the immune system as foreign antigens. With its limited depth of coverage (ranged $100 \mathrm{x}-200 \mathrm{x}$ ), WES might not have sufficient sensitivity for detecting variants at low-allele fraction, and might not be able to capture some of the mutations in realworld clinical samples with low tumor purity. In contrast, with the growing use of targeted NGS panels, more and more clinical trials have begun adopting panel-based TMB estimates (pTMB) as a stratification biomarker. ${ }^{4} 10$ The higher depth of coverage achieved by targeted NGS allows for the detection of variants at lower allelic fractions, enabling more sensitive detection of clonal variants in low-purity samples. ${ }^{8}$ Our premise is that measuring TMB with a small fraction of the exome with high depth of coverage using targeted deep sequencing (pTMB) can result in a better estimate of the true TMB than measuring TMB with the entire exome with low coverage (wTMB), particularly for clinical samples with low tumor purity. To evaluate this hypothesis, we reanalyzed the data of four publicly available ICI-treated cohort datasets. In addition, we examined an internal cohort profiled by paired NGS (WES and panel sequencing) to compare the clinical value of $\mathrm{PTMB}$ and $\mathrm{wTMB}$.

\section{METHODS \\ Study design and cohort characteristics}

This study was divided into three separate analyzes (figure 1A), including identification of the effects of tumor purity in the predictive performance of TMB from public data, generation of paired-NGS cohorts and clinical validation. The distribution of the tumor purity estimates across clinical NGS samples was investigated in two datasets of a targeted sequencing panel, CancerSCAN ${ }^{11}$ $(\mathrm{n}=6017)$ and Memorial Sloan Kettering-Integrated Mutation Profiling of Actionable Cancer Targets ${ }^{12}$ (MSKIMPACT, $\mathrm{n}=10$ 475), which was compared with that of The Cancer Genome Atlas (TCGA) samples ${ }^{13}(\mathrm{n}=9364)$. In addition, survival data of public cohorts ${ }^{18914}$ treated with immunotherapy were re-analyzed with respect to the efficacy of the two biomarkers in the context of low tumor purity. A total of four public cohorts were classified based on the type of biomarker (wTMB or pTMB) used. The 'External-WES' cohort comprised 195 ICI-treated patients with non-small-cell lung cancer (NSCLC) profiled with WES from three cohorts, ${ }^{8914}$ and the 'External-Panel' cohort comprised 1089 ICI-treated patients profiled with panel sequencing. ${ }^{1}$ Additional information on data acquisition is described in online supplemental data 1 .

From June 2014 to April 2019, a total of 279 NSCLC patients treated with antiprogrammed death-1/ programmed death ligand-1 (PD-L1) agents were profiled with $\mathrm{WES}^{15}$ at our institution. Among them, 156 patients with available remaining material were additionally profiled with CancerSCAN ('paired-NSCLC cohort') for this analysis. The same tumor sample was used for both panel sequencing and WES in all (100\%) patients; 144 $(92.3 \%)$ were from the same DNA aliquot. In addition, a dataset containing a total of 165 patients with breast cancer with paired NGS data ('paired-BRCA cohort') was also generated in the same manner. ${ }^{16}$ Detailed information of the patient cohorts is presented in online supplemental table S1 and S2.

Patients in the paired-NSCLC cohort with data for the response to ICI treatment and survival were further analyzed to validate the clinical value of pTMB in parallel with wTMB according to tumor purity. All patients provided informed consent for sample acquisition for research purposes.

\section{Assessment of response to ICI treatment}

For patients that received ICI treatment, the objective response was assessed by physicians using the Response Evaluation Criteria in Solid Tumors V.1.1. Patients showing a complete response or partial response were defined as responders, whereas patients with stable disease or progressive disease were classified as non-responders. The objective response rate (ORR) was defined as the proportion of responders. Progression-free survival (PFS) was calculated from the start date of ICI treatment to the date of progression or death.

\section{NGS workflow}

Targeted NGS was performed using CancerSCAN V.2, a tumor-only, targeted NGS platform designed at Samsung Medical Center with exonic regions of 381 cancerrelated genes $(1.07 \mathrm{Mb})$ and intronic regions of 23 genes in which fusion frequently occurs. ${ }^{11}$ DNA extraction, library preparation and raw data generation for WES and targeted NGS were conducted as previously described. ${ }^{11} 17$ Somatic variants and germline variants were detected by MuTect $2{ }^{18}$ and the GATK HaplotypeCaller, ${ }^{19}$ respectively. Sequencing errors and false-positive variants were manually curated using Integrative Genomics Viewer. The mean sequencing coverages of WES across all tumor samples and blood samples were $160 \times$ and $107 \times$, respectively. The mean coverage of targeted NGS across all tumor samples was $829 \times$. Details of the NGS workflow and variant detection are described in online supplemental data 1.

TMB and tumor purity estimation wTMB (expressed as mutations) was estimated as the total number of somatic non-synonymous single nucleotide variants (SNVs) and indels. The limit of detection (LOD) for wTMB estimation was set to a variant allele frequency (VAF) of $5 \%$. The pTMB (expressed as mutations/Mb) was estimated as the total number of somatic non-synonymous SNVs and indels divided by the covered coding region. The LOD for pTMB estimation was set to a VAF of $1 \%$. The subtraction of germline variants for targeted NGS was performed using the available germline databases, ${ }^{20-24}$ and its performance was assessed in the paired NGS dataset by comparing two types of pTMB (tumor-only vs tumor/matched normal, online supplemental figure S1). To evaluate the appropriateness of the panel size, correlations between the pairs of pTMB and wTMB were calculated by in silico simulations of stepwise alterations in the 
A

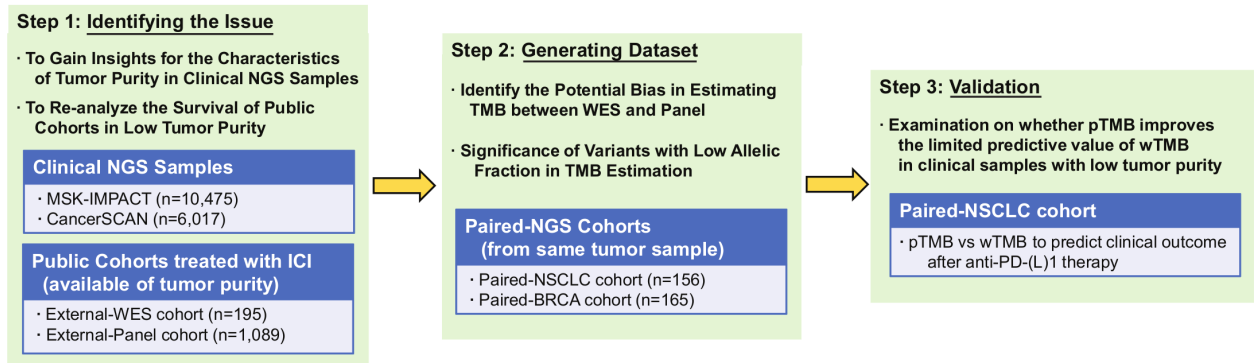

B

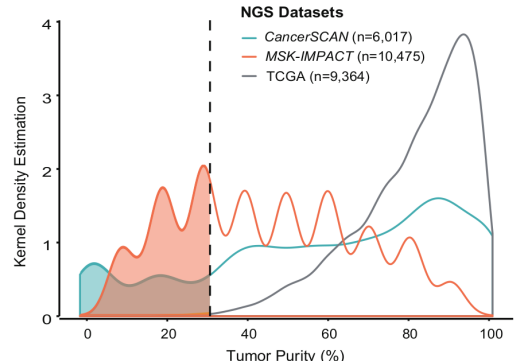

C
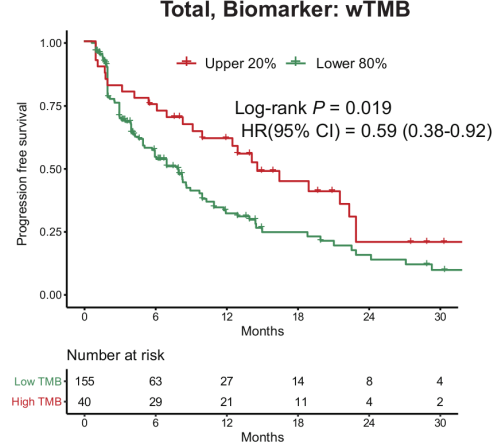

D

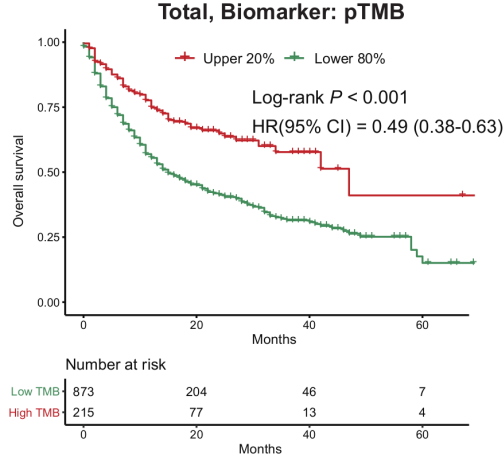

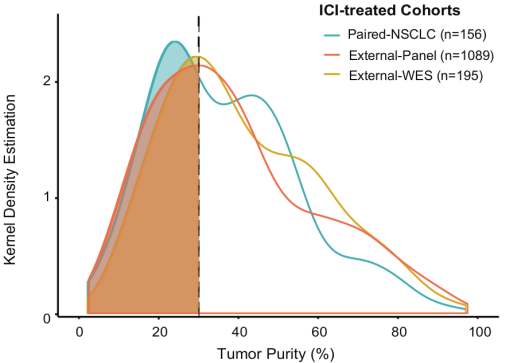

Low Purity $(\leq 30 \%)$, Biomarker: wTMB
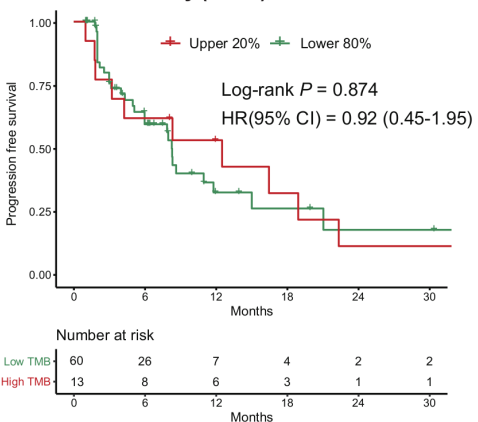

Low Purity $(\leq 30 \%)$, Biomarker: pTMB
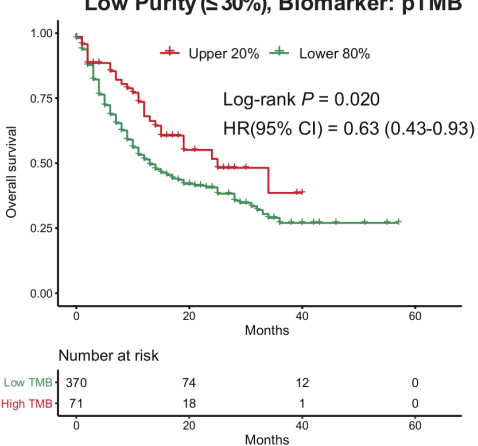

Figure 1 Study design, the distribution of tumor purity and reanalysis of public cohorts. (A) Study overview and cohort characteristics. (B) Distribution of tumor purity from two NGS datasets (CancerSCAN, MSK-IMPACT) and clinical cohorts treated with ICI (external-WES cohort, external-panel cohort and paired-NSCLC cohort of this study). (C) Influence of tumor purity on predictive performance of WES-based TMB in the External-WES cohort $(n=195)$. (D) Influence of tumor purity on predictive performance of panel-based TMB in the external-panel cohort $(n=1089)$. BRCA, breast cancer; ICl, immune checkpoint inhibitor; NGS, next-generation sequencing; NSCLC, non-small-cell lung cancer; pTMB, panel sequencing-based tumor mutational burden; SMC, Samsung Medical Center; TCGA, The Cancer Genome Atlas; TMB, tumor mutational burden; WES, whole-exome sequencing; wTMB, whole-exome sequencing-based tumor mutational burden.

number of included genes (online supplemental figure S2).

To control for the effect of interobserver variability on pathological determination of tumor purity, this analysis adopted computational purity metrics. In detail, tumor purity was determined on the basis of four computational algorithms: FACETS, ${ }^{25}$ Sequenza, ${ }^{26}$ PureCN $^{27}$ and a mutation-based estimation using the median VAF. Tumor 
purity was primarily inferred from FACETS according to the online manual. The other algorithms were prioritized based on their ability to reliably impute missing or unreliable values from FACETS (online supplemental figure S3A). For the analysis of targeted NGS data without WES, PureCN was implemented as the primary algorithm for tumor purity estimation. Manual curation of the results from each algorithm (eg, allele-specific copy number and ploidy levels) was performed in all cases, and details for determining tumor purity are presented in online supplemental figure S3B. For analyzes of external cohorts, TMB and tumor purity data were retrieved from the original publications. 18914

\section{PD-L1 expression and tumor-infiltrating lymphocytes analysis}

Immunohistochemistry of PD-L1 was performed using the Dako PD-L1 IHC 22C3 pharmDx kit (Agilent Technologies, Santa Clara, California, USA). PD-L1 expression scores were reported as the proportion of stained tumor cells, as determined by the thoracic pathologists. PD-L1 subgroups were stratified based on low $(0 \%-49 \%)$ and high $(\geq 50 \%)$ expression.

Tumor-infiltrating lymphocyte (TIL) was assessed under $\mathrm{H} \& \mathrm{E}$ sections by two independent thoracic pathologists to control the confounding effect of TIL on outcomes. It was reported as continuous variable (proportion score) according to the current pathological guideline. ${ }^{28}$

\section{Cut-off points and statistical analysis}

The samples were stratified into two groups based on the TMB (high vs low) using the top $20 \%$ as the cut-off point. ${ }^{1}$ The cut-off point for tumor purity was set to $30 \%$, based on the degree of the purity-related bias in TMB estimates (see online supplemental data 1 and online supplemental figure S4). Details of statistical analysis are described in online supplemental data 1 .

\section{RESULTS}

\section{Characteristics of tumor purity and pTMB in real-world datasets}

All analyzed ICI-treated cohorts, as well as the two targeted NGS datasets (CancerSCAN and MSK-IMPACT), showed even distributions of tumor purity, whereas TCGA samples exhibited a skewed distribution with an overrepresentation of high tumor purity (figure 1B). Samples with low tumor purity accounted for $30.4 \%$ of all analyzed samples (4993 of 16551 samples) across the two real-world NGS datasets, whereas the TCGA cohort rarely contained samples with low purity (8 out of 9364 included samples, $0.1 \% ; \mathrm{p}<0.001)$. In ICI-treated cohorts, patients with tumor samples of low purity accounted for $40.5 \%$ of all patients (583 of 1440 patients; range, 30.3\%-44.9\%).

The distribution of pTMB from the two datasets was similar across cancer types and was consistent with previous results for cancer types with high and low mutation rates $^{29}$ (online supplemental figure S5A). However, the pTMB for CancerSCAN was higher than that for
MSK-IMPACT (median difference, 3.1 mutations/Mb). The germline components that passed the common single nucleotide polymorphism filter (median, 2.9 mutations $/ \mathrm{Mb}$ ) in the tumor-only pipeline largely explained the observed difference in pTMB between the two datasets (online supplemental figure S5B).

\section{Reanalyses of survival outcomes in public cohorts treated with ICI}

For the External-WES cohort ( $\mathrm{n}=195)$, wTMB-based survival outcome stratification was effective (log-rank $p=0.019$, left side of figure 1C). However, wTMB failed to predict PFS in the low-purity subgroup (log-rank $\mathrm{p}=0.874$, right side of figure $1 \mathrm{C})$. For the external-panel cohort $(n=1089)$, the predictive performance of pTMB was high (log-rank $p<0.001$, left side of figure $1 \mathrm{D})$, even for the low-purity subgroup ( $\log$-rank $\mathrm{p}=0.020$, right side of figure 1D).

\section{Comparative analysis of the effect of tumor purity on pTMB and wTMB estimates}

Using paired NGS data (ie, pTMB and wTMB for the same tumor specimen), we evaluated the effect of tumor purity on TMB estimates and their correlation. A linear regression analysis showed that tumor purity significantly influences wTMB $(p<0.001)$ but not pTMB $(p=0.513$, left side of figure 2A). These results were reproducible in the paired-BRCA cohort (right side of figure 2A).

In the paired-NSCLC cohort, there was substantial disagreement in TMB groups between the two platforms (positive percent agreement $(\mathrm{PPA})=64.5 \%$, online supplemental table S3). This discrepancy was more prominent in the low-purity subgroup (PPA for adequate vs low-purity subgroups, $68.5 \%$ vs $58.3 \%$ ), suggesting that there is a serious risk of misclassification in samples with low purity.

\section{More sensitive detection of low-VAF variants in panel sequencing}

Next, we investigated mechanistic factors related to the difference in the susceptibility of each platform with respect to tumor purity. In the paired cohort of NSCLC and BRCA, the somatic variants detected by panel sequencing were characterized and visualized based on their VAF (figure 2B). The percentages of variants not detected by WES was much higher in the low-VAF region $(\mathrm{p}<0.001)$. More importantly, the proportion of low-VAF variants not detected by WES was increased in low-purity samples compared with that in high-purity samples $(23 \%$ vs $15 \%, \mathrm{p}<0.001)$. To explore the influence of tumor purity in the VAF of clonal variants, the VAF distribution of hotspot SNVs was analyzed in 16492 clinical samples across the two targeted NGS datasets, CancerSCAN and MSK-IMPACT (figure 3). Compared with high-purity samples, the low-purity samples presented a larger proportion of hotspot variants with low VAF less than $10 \%$ ( $40.4 \%$ vs $17.0 \%, \mathrm{p}<0.001$, figure $3 \mathrm{~B})$. This result suggests that low-VAF variants, which are primarily detected by 


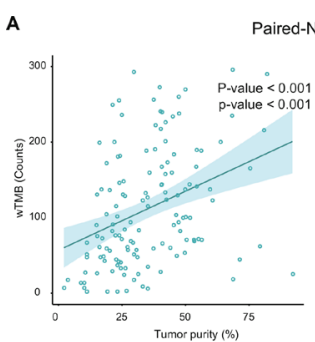

B
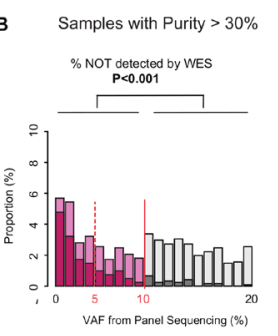

口qDetected by WES
aired-NSCLC ( $n=156)$

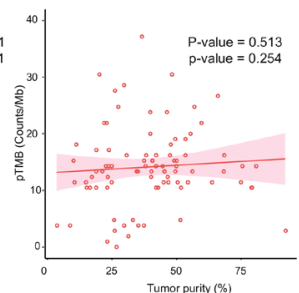

Samples with Purity $\leq 30 \%$ $\%$ NOT detomed D WES

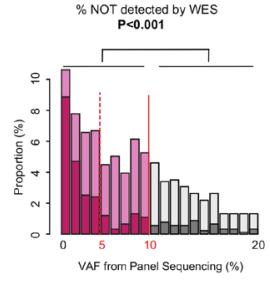

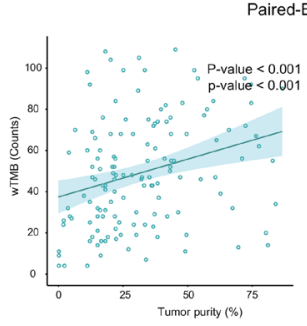

c

Paired-NSCLC ( $n=156)$

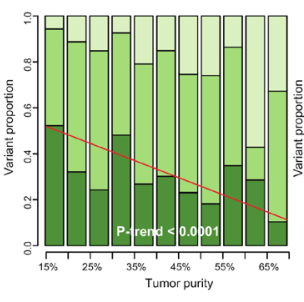

Paired-BRCA (n=165)

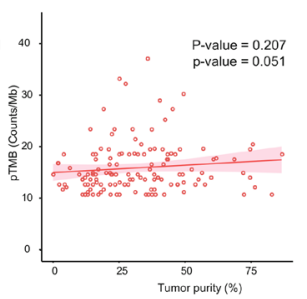

Paired-BRCA ( $n=165)$

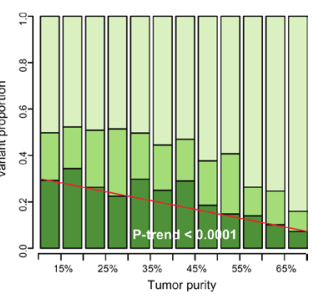

口 Exclusively Detected (WES) a Concordantly Detected a Exdusively Detected (Panel-Seq)

Figure 2 Differential impacts of tumor purity on the two biomarkers. (A) Impact of tumor purity on two types of biomarkers identified from the linear regression. Left: paired-NSCLC cohort; right: paired-BRCA cohort. (B) Prevalence of variants with a low allele fraction. Red lines at $5 \%$ and $10 \%$ indicate common cut-off points for VAF in pTMB estimation. (C) Trend in the configuration of platforms (by which variants were detected) under changes of tumor purity. BRCA, breast cancer; NSCLC, nonsmall-cell lung cancer; pTMB, panel sequencing-based tumor mutational burden; VAF, variant allele frequency; WES, wholeexome sequencing; wTMB, whole-exome sequencing-based tumor mutational burden.

A

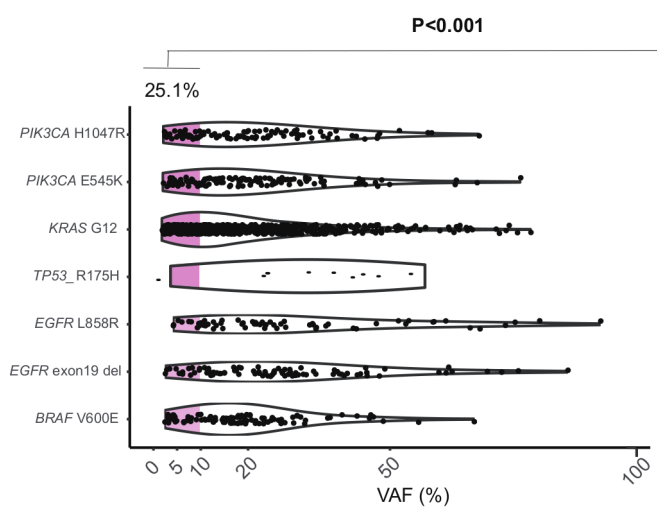

B

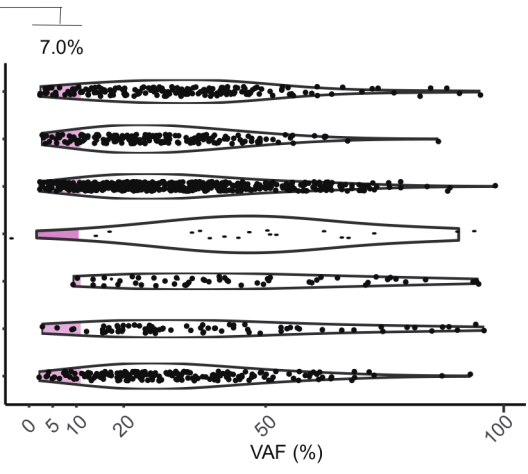

CancerSCAN, Adequate purity
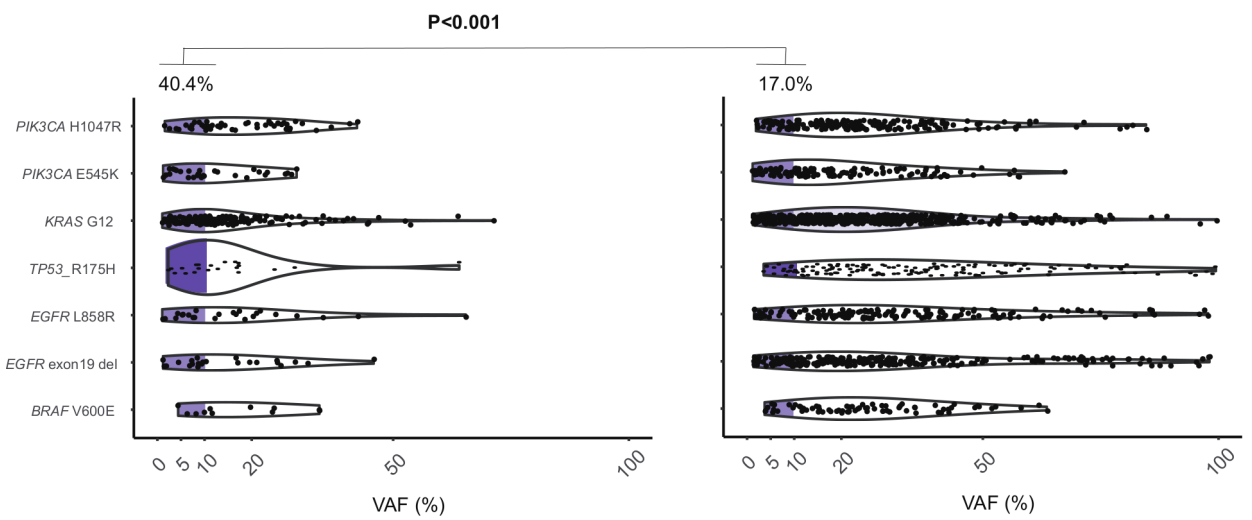

Figure 3 Prevalence of SNVs and Indels with low VAF according to the tumor purity groups from the two NGS datasets. (A) MSK-IMPACT ( $n=10475)$. (B) CancerSCAN ( $n=6017)$. Left, adequate purity $(>30 \%)$ samples, right: low-purity samples. NGS, next-generation sequencing; SNV, single-nucleotide variant; VAF, variant allele frequency. 
panel sequencing, can arise not only from subclonal variants but also from clonal variants in low-purity samples.

To confirm the higher sensitivity of targeted NGS as compared with WES, somatic variants of the pairedNSCLC cohort were classified on the basis of the NGS platform on which they were detected. Astoundingly, the proportion of variants detected exclusively by targeted NGS tended to increase as tumor purity decreased ( $p$ trend $<0.001$, left side of figure $2 \mathrm{C}$ ). This trend was reproducible in the paired-BRCA cohort ( $p$ trend $<0.001$, right side of figure $2 \mathrm{C}$ ).

\section{Superior clinical performance of pTMB at predicting the benefits of anti-PD-(L)1 therapy in samples with low tumor purity}

The paired-NSCLC cohort was further scrutinized to study the association between purity-related bias on TMB and impaired prediction for the ICI response. Figure 3A summarizes the clinicogenomic characteristics for 156 patients. Patients correctly reclassified using pTMB were enriched in the low-purity subgroup (black arrows, figure $4 \mathrm{~A}$ ).

Regarding the ORR, both wTMB and pTMB effectively discriminated responders from non-responders among all paired-NSCLC patients (wTMB: $48.4 \%$ vs $21.6 \%$, $\mathrm{p}=0.006$; pTMB: $58.1 \%$ vs $19.2 \%, \mathrm{p}<0.001$, figure $4 \mathrm{~B})$. In the subgroup analysis of the low-purity group $(n=69)$, wTMB could not sufficiently discriminate responders with borderline significance $(66.7 \%$ vs $27.4 \%, \mathrm{p}=0.050)$, whereas pTMB had superior predictive ability $(83.3 \%$ vs $22.0 \%, p<0.001$, figure 4B). pTMB had higher accuracy than wTMB for predicting the response (area under the curve of 0.61 for wTMB and 0.70 for pTMB). The net

A
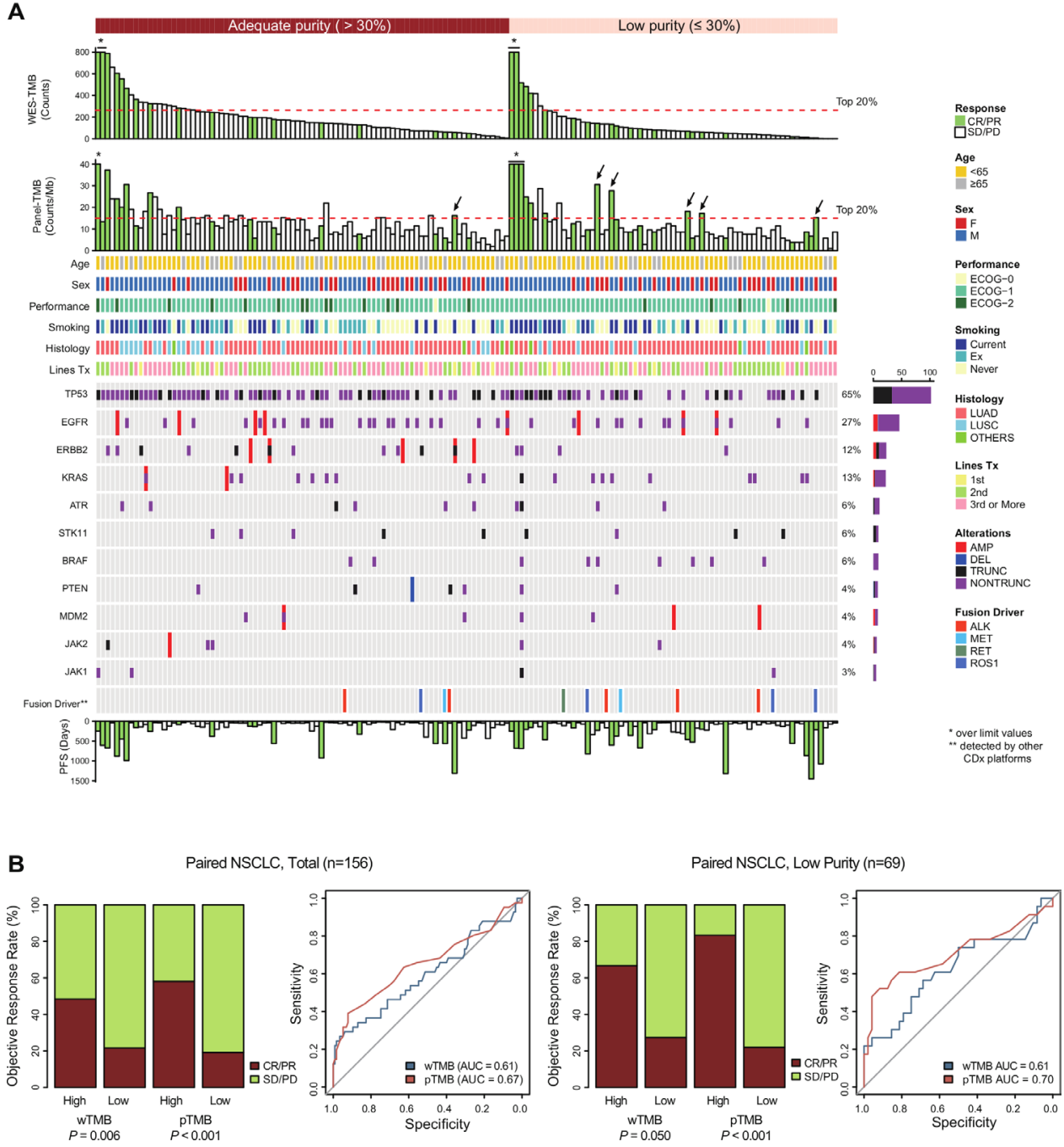

Figure 4 Clinical and genomic characteristics of the paired-NSCLC cohort and responder prediction using the two biomarkers. (A) Heatmap illustrating the clinical and genomic data of 156 patients in the paired-NSCLC cohort. Arrows indicate correctly reclassified patients using pTMB. (B) Comparison of the two biomarkers (wTMB vs pTMB) in the objective response rate and ROC curve analysis (left side: total patients, right side: patients with low purity). AUC, area under the curve; CDx, companion diagnostics; ECOG, Eastern Cooperative Oncology Group; NSCLC, non-small-cell lung cancer; LUAD, lung adenocarcinoma; LUSC, lung squamous cell carcinoma; PFS, progression-free survival; pTMB, panel sequencing-based tumor mutational burden; ROC, receiver operating characteristic; WES, whole-exome sequencing; wTMB, whole-exome sequencing-based tumor mutational burden. 
A
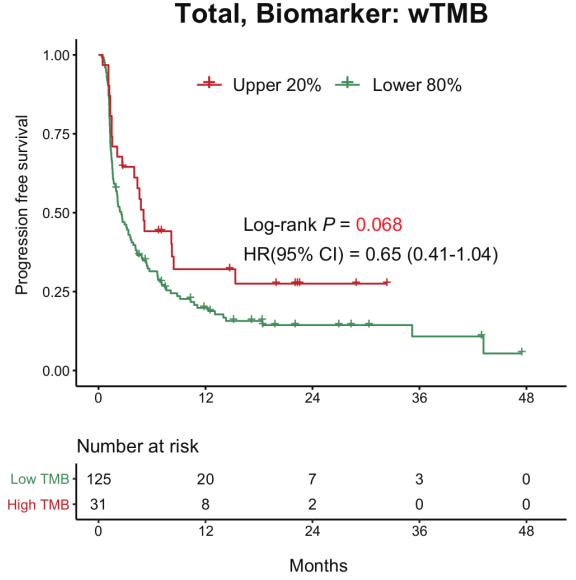

B

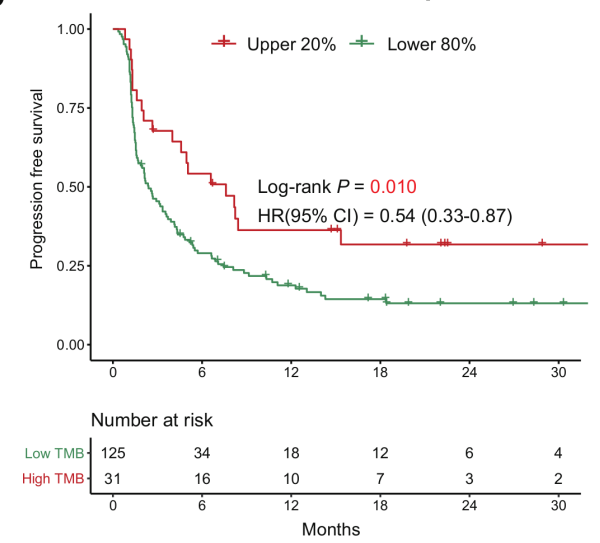

Low Purity ( $\leq 30 \%)$, Biomarker: wTMB
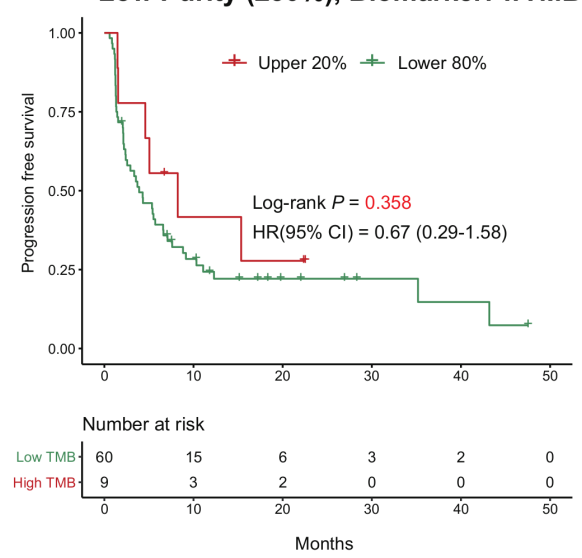

Low Purity ( $\leq 30 \%)$, Biomarker: pTMB
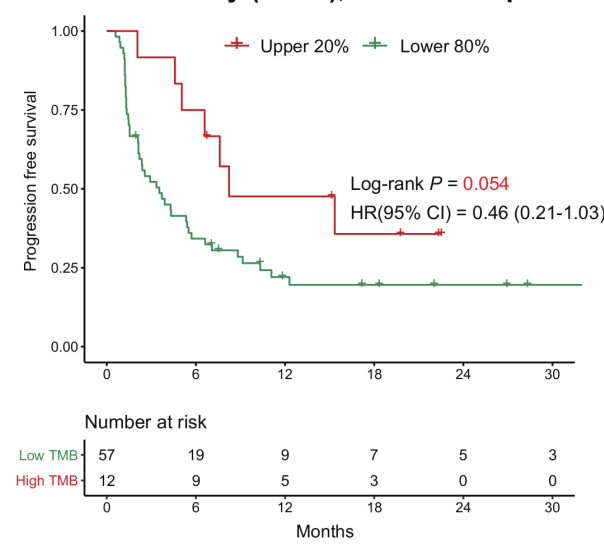

Figure 5 Survival analysis of the paired-NSCLC cohort treated with ICl using the two biomarkers. (A) The use of wTMB as a stratification biomarker (left side: total patients, right side: patients with low-purity samples). (B) The use of pTMB as a stratification biomarker. (Left side: total patients, right side: patients with low-purity samples). ICl, immune checkpoint inhibitor; pTMB, panel sequencing-based tumor mutational burden; wTMB, whole-exome sequencing-based tumor mutational burden.

reclassification in the low-purity subgroup was significantly improved on using pTMB instead of wTMB (categorical net classification improvement: $21.7 \%$, 95\% CI $4.1 \%$ to $39.4 \%, \mathrm{p}=0.016)$. The performance of $\mathrm{pTMB}$ as a stratification biomarker was also superior than wTMB when the effects of immune cell infiltration is controlled (online supplemental figure S6).

In terms of survival outcome, a high wTMB tended to stratify patients based on PFS with borderline significance $(p=0.068)$, but could not predict PFS in the subgroup with low purity (HR $0.67, \mathrm{p}=0.358$, figure $5 \mathrm{~A}$ ). Interestingly, a high pTMB not only predicted longer PFS in all patients (median PFS, 7.61 months vs 2.39 months, HR $0.54, \mathrm{p}=0.010$ ) but also provided improved prediction for PFS in the subgroup with low purity (median PFS, 8.23 months vs 3.54 months, HR 0.46, $\mathrm{p}=0.054$, figure $5 \mathrm{~B})$. In terms of overall survival (OS), the use of pTMB tends to work better in the OS stratification compared with the use of wTMB though it lacked statistical significance (online supplemental figure S7). After adjusting for age, sex, performance status, and lines of treatment, pTMB was consistently identified as an independent predictor of PFS across all purity groups (low-purity group; adjusted HR $0.32,95 \%$ CI 0.21 to $1.41, \mathrm{p}=0.016$, table 1$)$. In contrast, the predictive value of WTMB in the multivariate model was substantially reduced in the low-purity subgroup (adjusted HR 0.54, $95 \%$ CI 0.21 to $1.41, p=0.212$, table 1 ). Sensitivity analysis including PD-L1 expression as a covariate demonstrated the superiority of pTMB over wTMB for predicting the ICI response in the low-purity subgroup (online supplemental table S4).

To demonstrate the robustness of our analysis in the setting of different thresholds for tumor purity and TMB, we performed several sensitivity analyzes. In accordance with previous results, pTMB tended to act as a better stratification biomarker than wTMB in the setting of different definitions for low tumor purity $(<25 \%$ and $<35 \%$, online supplemental figure S8). In addition, when TMB was considered as a continuous variable rather than selecting the top $20 \%$ as a cut-off, we observed that pTMB was consistently identified as an independent predictor of PFS across all purity groups (low-purity group; adjusted HR $0.75,95 \%$ CI 0.58 to $0.96, p=0.021$ ) while wTMB was not (low-purity group; adjusted HR 0.96, 95\% CI 0.82 to 1.10, $\mathrm{p}=0.572$; Online supplemental figure S9). 


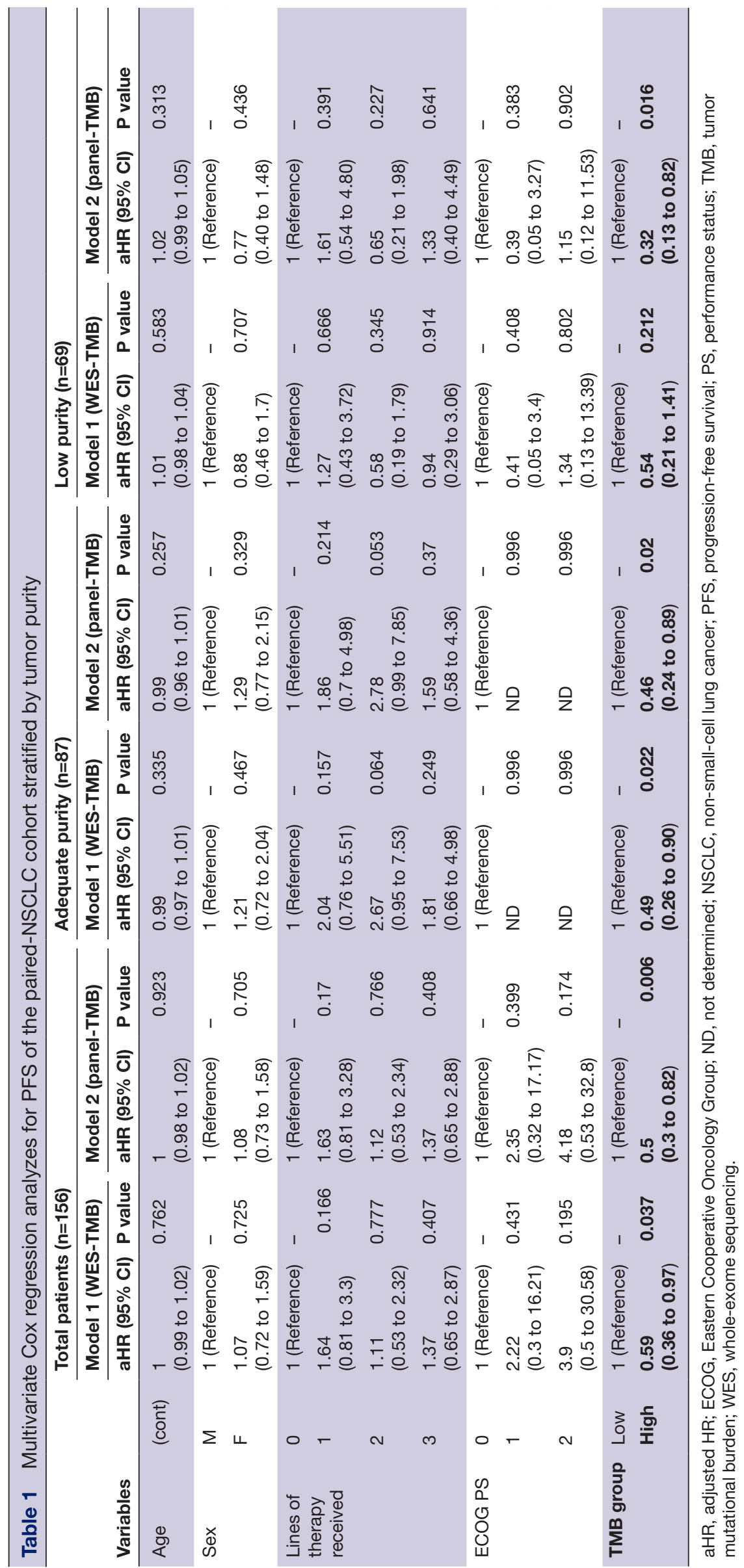

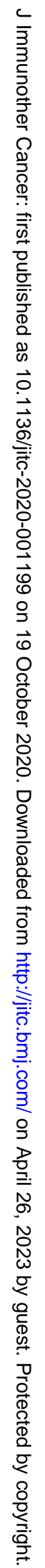




\section{DISCUSSION}

To our knowledge, this study represents the largest series of WES-targeted NGS paired datasets analyzed to date to determine whether targeted NGS could more accurately estimate TMB, even for a high proportion of samples with low tumor purity. Several studies ${ }^{90}$ have indicated the feasibility of pTMB to predict clinical benefits from immunotherapy with good correlations against wTMB; however, there has been no investigation on whether pTMB overcomes the limitation of wTMB for predictions with low-purity samples. Minimizing the impact of tumor purity on TMB estimates has implications for the interpretation of trial results and for individual patient management outside of clinical trials. ${ }^{31}$

Our analysis is grounded on the importance of lowpurity samples in real-world clinical settings ${ }^{11}$ and the increased sensitivity of targeted NGS for scant variant detection. To address this complex issue, we used a threestep approach: (1) reanalysis of survival in public ICItreated cohorts with real-world characteristics of tumor purity in clinical samples evaluated by NGS, (2) analysis of the relative susceptibility of each biomarker to purityrelated bias and its mechanistic explanation and (3) comparative analysis of the predictive value of WTMB and pTMB for low-purity samples.

The observed consistency of pTMB distributions for two datasets (CancerSCAN and MSK-IMPACT) could reflect the reliability of both targeted NGS platforms. Several factors impact the reliability of pTMB, including the size $^{32} 33$ (whether the panel covers a sufficient area of the genome), correlation (between pTMB and wTMB), and the filtering algorithms used for germline variants. ${ }^{34} 35$ We controlled for the aforementioned factors to ensure the validity of pTMB assessed by CancerSCAN. Although harmonization across various targeted NGS panels is still required, evidence for the validity of targeted NGSbased TMB estimation across various tumor types is accumulating. ${ }^{6123036}$ Of note, the QuIP study ${ }^{6}$ recently compared seven commercial targeted NGS panels and demonstrated the general reliability of pTMB estimates, regardless of panel type.

In particular, the impact of germline variants on TMB estimates appears to be non-negligible. WES always uses matched germline analysis and is free of this issue; however, as we demonstrated, wTMB has weak points in low tumor purity settings due to the limited depth of coverage, which might pose a bigger problem. As for panel sequencing, both tumor-only (eg, Foundation, CancerSCAN) and matched germline analysis (eg, MSKIMPACT) can be used. In NGS panels adopting a tumoronly approach, unfiltered germline variants can affect the TMB estimates. In these cases, the absolute value may be falsely overestimated, resulting in a slight increase in the overall TMB. However, applying a rank-based cutoff point, along with harmonization across other panelbased platforms, would alleviate this bias. In addition, it is urgent to develop/apply an algorithm that can more thoroughly remove germline variants in tumor-only sequencing methods.

Our preliminary analysis also sheds light on the realworld distribution of tumor purity in clinical samples profiled by targeted NGS. It is well known that TCGA excludes $>50 \%$ of all samples submitted based on tumor purity $(<60 \%) \cdot{ }^{713}$ However, it is noteworthy that samples with low purity $(<30 \%)$ accounted for a significant portion of the real-world samples from both large NGS datasets and ICI-treated cohorts. Accordingly, the unsatisfactory performance of wTMB in patients with low tumor purity is a serious hurdle for the future application of TMB in precision medicine.

We observed that targeted NGS is highly sensitive for the detection of variants at low allelic fractions. According to a previous study, ${ }^{11}$ usual depth of coverage in WES (150 $\mathrm{x}-200 \mathrm{x}$ ) showed a sensitivity of only about $40 \%$ for variants with $5 \%$ VAF; however, panel sequencing offers depth of coverage typically ranging around $800 \mathrm{x}$ or more, which can detect more than $99 \%$ of low-VAF $(<5 \%)$ variants. Taken together, our results might imply the clinical need for deep sequencing with a lower LOD for accurate TMB estimation. Nevertheless, further studies are required to determine the ideal LOD (eg, VAF under $5 \%$ or $1 \%$ ) for estimating pTMB considering the risk of including falsepositive reads arising from deamination artifacts and subclonal variants with uncertain immunogenicity, as well as the need for the sensitive detection of clonal variants in low-purity samples. Our result also has an implication for expanding the limited evaluability in TMB estimation, which remains a major concern for implementing tissuebased TMB as a biomarker for patient selection. Even when the sample's purity used for TMB analysis is low $(<10 \%-20 \%)$, patients who might have been excluded from the trials adopting wTMB can be evaluated for accurate TMB estimation with greater sensitivity of a targeted NGS panel.

Ultimately, our main aim was to assess whether the predictive power of WTMB $^{\text {in }}$ clinical samples with low tumor purity can be improved using pTMB. The uniqueness of paired NGS data along with ICI responses enabled us to comprehensively compare TMB estimates from each NGS platform. We can reasonably judge that purity-related misprediction is infrequent in previous and ongoing clinical trials using pTMB.

Our data also challenge conventional notions about TMB. To be specific, WES-based TMB estimates should be interpreted with caution when there are many samples with low purity $(<30 \%)$, suggesting that WES should be reconsidered as the gold standard. Despite reflection of the total neoantigen burden by definition, the reliability of wTMB as a biomarker in low-purity samples is uncertain owing to its limited ability to effectively call somatic variants with low frequency. At the same time, our study emphasizes the accuracy of targeted NGS, especially for clinicians who have long-standing doubts regarding this approach. Considering other advantages of panel sequencing (eg, reduced cost, expanded tissue 
availability and shorter turn-around time), our data provide important insight into the robustness of pTMB for clinical use, and may facilitate the use of pTMB as a relevant biomarker for the immunotherapy response.

Our study had the following limitations. First, our survival analyzes were primarily based on PFS instead of OS. The use of PFS as a surrogate for OS is considered an inherent limitation of small-sized biomarker studies using TMB and should be addressed by large-scale prospective trials. Second, despite including the largest series of cohorts with paired NGS data to date, our analysis still had insufficient statistical power. For a sufficient number of low-purity samples, paired NGS data should be generated for a greater number of patients, which may be unrealistic. Finally, there were 12 (7.7\%) NSCLC patients whose DNA aliquot was not identically used for sequencing. However, even in those cases, the DNA was extracted from the same tissue block. Moreover, this bias is unlikely to be large since the same DNA was used in most (>90\%) patients.

In conclusion, our study suggests an advantage in the use of panel sequencing-based TMB for low-purity samples. Targeted panel sequencing provides reliable predictive performance for the response to anti-PD-(L) 1 therapy by mitigating the bias from low purity. Clinical use of pTMB can be encouraged for both clinical trials and real-world settings which frequently involve samples with low tumor purity. Since our findings were limited by the small number of patients included, further validation of our findings is warranted in larger cohorts.

\section{Author affiliations \\ ${ }^{1}$ Samsung Genome Institute, Samsung Medical Center, Seoul, Korea \\ ${ }^{2}$ Department of Digital Health, Samsung Advanced Institute of Health Science and Technology, Sungkyunkwan University, Seoul, Korea \\ ${ }^{3}$ Division of Hematology-Oncology, Department of Medicine, Samsung Medical Center, Sungkyunkwan University School of Medicine, Seoul, Korea ${ }^{4}$ Department of Health Science and Technology, Samsung Advanced Institute of Health Science and Technology, Sungkyunkwan University, Seoul, Korea ${ }^{5}$ GENINUS Inc, Seoul, Korea \\ ${ }^{6}$ Department of Pathology and Translational Medicine, Samsung Medical Center, Sungkyunkwan University School of Medicine, Seoul, Korea \\ ${ }^{7}$ Department of Molecular Cell Biology, Sungkyunkwan University School of Medicine, Suwon, Korea}

Acknowledgements We would like to acknowledge all the patients and their families for their contributions to this study.

Contributors $\mathrm{THH}$ and $\mathrm{HC}$ conceived the experimental design, performed data analysis and drafted the manuscript. $\mathrm{THH}$ and $\mathrm{HC}$ refined the experimental design and performed data analysis. $\mathrm{HC}$ and BL performed data analysis. SP, S-HL and $\mathrm{W}-\mathrm{YP}$ refined experimental design and edited the manuscript. All authors have read and approved the manuscript.

Funding This work was supported by the Korean Health Technology R\&D Project, Ministry of Health and Welfare, Republic of Korea (H13C2096 to W-YP), the National Research Foundation of Korea (NRF) grant funded by the Korea government (MSIT) (2020R1A2C3006535 to S-HL), and the National Cancer Center Grant (NCC1911269-2 to S-HL).

Competing interests JSA reports personal fees from Amgen, personal fees from Pfizer, personal fees from AstraZeneca, personal fees from Menarini, personal fees from Roche, personal fees from Eisai, personal fees from Boehringer Ingelheim, personal fees from BMS-Ono, personal fees from MSD, personal fees from Janssen, personal fees from Samsung Bioepis, outside the submitted work. S-HL reports grants and personal fees from MSD, personal fees from Novartis, personal fees from AstraZeneca, personal fees from BMS, personal fees from Roche, outside the submitted work. Keunchil Park reports personal fees from Astellas, Astra Zeneca, AMGEN, Boehringer Ingelheim, Clovis, Eli lilly, Hanmi, KHK, Merck, MSD, Novartis, ONO, Roche, BluePrint, outside the submitted work. SP reports stock holding of Lunit. W-YP, CL and NKDK are employed by a commercial company, GENINUS. All remaining authors have declared no conflicts of interest.

Patient consent for publication Not required.

Ethics approval The study was conducted under an institutional review boardapproved protocol (IRB No. 2013-04-005, 2012-08-065).

Provenance and peer review Not commissioned; externally peer reviewed.

Data availability statement Data are available on reasonable request. All data relevant to the study are included in the article or uploaded as online supplemental information. All data and materials relevant to this article are available to referees at submission and to readers promptly on request.

Supplemental material This content has been supplied by the author(s). It has not been vetted by BMJ Publishing Group Limited (BMJ) and may not have been peer-reviewed. Any opinions or recommendations discussed are solely those of the author(s) and are not endorsed by BMJ. BMJ disclaims all liability and responsibility arising from any reliance placed on the content. Where the content includes any translated material, BMJ does not warrant the accuracy and reliability of the translations (including but not limited to local regulations, clinical guidelines, terminology, drug names and drug dosages), and is not responsible for any error and/or omissions arising from translation and adaptation or otherwise.

Open access This is an open access article distributed in accordance with the Creative Commons Attribution 4.0 Unported (CC BY 4.0) license, which permits others to copy, redistribute, remix, transform and build upon this work for any purpose, provided the original work is properly cited, a link to the licence is given, and indication of whether changes were made. See https://creativecommons.org/ licenses/by/4.0/.

\section{ORCID iD}

Tae Hee Hong http://orcid.org/0000-0003-2708-8893

\section{REFERENCES}

1 Samstein RM, Lee C-H, Shoushtari AN, et al. Tumor mutational load predicts survival after immunotherapy across multiple cancer types. Nat Genet 2019;51:202-6.

2 Hellmann MD, Nathanson T, Rizvi H, et al. Genomic features of response to combination immunotherapy in patients with advanced non-small-cell lung cancer. Cancer Cell 2018;33:843-52.

3 Keenan TE, Burke KP, Van Allen EM. Genomic correlates of response to immune checkpoint blockade. Nat Med 2019;25:389-402.

4 Chan TA, Yarchoan M, Jaffee E, et al. Development of tumor mutation burden as an immunotherapy biomarker: utility for the oncology clinic. Ann Oncol 2019;30:44-56.

5 Büttner R, Longshore JW, López-Ríos F, et al. Implementing TMB measurement in clinical practice: considerations on assay requirements. ESMO Open 2019;4:e000442-12.

6 Stenzinger A, Endris V, Budczies J, et al. Harmonization and Standardization of Panel-Based Tumor Mutational Burden Measurement: Real-World Results and Recommendations of the Quality in Pathology Study. J Thorac Oncol 2020;15:1177-89.

7 Patel NM, Jo H, Eberhard DA, et al. Improved tumor purity metrics in next-generation sequencing for clinical practice. Appl Immunohistochem Mol Morphol 2019;27:764-72.

8 Anagnostou V, Niknafs N, Marrone K, et al. Multimodal genomic features predict outcome of immune checkpoint blockade in nonsmall-cell lung cancer. Nat Cancer 2020;1:99-111.

9 Rizvi H, Sanchez-Vega F, La K, et al. Molecular determinants of response to anti-programmed cell death (PD)-1 and antiprogrammed death-ligand 1 (PD-L1) blockade in patients with non-small-cell lung cancer profiled with targeted next-generation sequencing. J Clin Oncol 2018;36:633-41.

10 Greillier L, Tomasini P, Barlesi F. The clinical utility of tumor mutational burden in non-small cell lung cancer. Transl Lung Cancer Res 2018;7:639-46.

11 Shin H-T, Choi Y-L, Yun JW, et al. Prevalence and detection of low-allele-fraction variants in clinical cancer samples. Nat Commun 2017;8:1-10.

12 Zehir A, Benayed R, Shah RH, et al. Mutational landscape of metastatic cancer revealed from prospective clinical sequencing of 10,000 patients. Nat Med 2017;23:703-13. 
13 Aran D, Sirota M, Butte AJ. Systematic pan-cancer analysis of tumour purity. Nat Commun 2015;6:8971.

14 Miao D, Margolis CA, Vokes NI, et al. Genomic correlates of response to immune checkpoint blockade in microsatellite-stable solid tumors. Nat Genet 2018;50:1271-81.

15 Shim JH, Kim HS, Cha H, et al. HLA-corrected tumor mutation burden and homologous recombination deficiency for the prediction of response to PD-(L)1 blockade in advanced non-small-cell lung cancer patients. Ann Oncol 2020;31:902-11.

16 Kan Z, Ding Y, Kim J, et al. Multi-Omics profiling of younger Asian breast cancers reveals distinctive molecular signatures. Nat Commun 2018:9:1-13.

17 Kim K, Kim HS, Kim JY, et al. Predicting clinical benefit of immunotherapy by antigenic or functional mutations affecting tumour immunogenicity. Nat Commun 2020;11:1-11.

18 Cibulskis K, Lawrence MS, Carter SL, et al. Sensitive detection of somatic point mutations in impure and heterogeneous cancer samples. Nat Biotechnol 2013;31:213-9.

19 DePristo MA, Banks E, Poplin R, et al. A framework for variation discovery and genotyping using next-generation DNA sequencing data. Nat Genet 2011;43:491-8.

20 , Auton A, Brooks LD, Abecasis GR, et al, 1000 Genomes Project Consortium. A global reference for human genetic variation. Nature 2015;526:68-74.

21 Tennessen JA, Bigham AW, O'Connor TD, et al. Evolution and functional impact of rare coding variation from deep sequencing of human exomes. Science 2012;337:64-9.

22 Lek M, Karczewski KJ, Minikel EV, et al. Analysis of protein-coding genetic variation in 60,706 humans. Nature 2016;536:285-91.

23 Karczewski KJ, Francioli LC, Tiao G, et al. The mutational constraint spectrum quantified from variation in 141. 456 humans. bioRxiv 2019;531210.

24 Korean reference genome database (KRGDB).

25 Shen R, Seshan VE. Facets: allele-specific copy number and clonal heterogeneity analysis tool for high-throughput DNA sequencing. Nucleic Acids Res 2016;44:e131-9.
26 Favero F, Joshi T, Marquard AM, et al. Sequenza: allele-specific copy number and mutation profiles from tumor sequencing data. Ann Oncol 2015;26:64-70.

27 Riester M, Singh AP, Brannon AR, et al. PureCN: copy number calling and SNV classification using targeted short read sequencing. Source Code Biol Med 2016;11:1-13.

28 Hendry S, Salgado R, Gevaert T, et al. Assessing tumor-infiltrating lymphocytes in solid tumors. Adv Anat Pathol 2017;24:235-51.

29 Alexandrov LB, Nik-Zainal S, Wedge DC, et al. Signatures of mutational processes in human cancer. Nature 2013;500:415-21.

30 Chalmers ZR, Connelly CF, Fabrizio D, et al. Analysis of 100,000 human cancer genomes reveals the landscape of tumor mutational burden. Genome Med 2017:9:34.

31 Budczies J, Allgäuer M, Litchfield K, et al. Optimizing panelbased tumor mutational burden (TMB) measurement. Ann Oncol 2019;30:1496-506.

32 Garofalo A, Sholl L, Reardon B, et al. The impact of tumor profiling approaches and genomic data strategies for cancer precision medicine. Genome Med 2016;8:79.

33 Buchhalter I, Rempel E, Endris V, et al. Size matters: dissecting key parameters for panel-based tumor mutational burden analysis. Int $J$ Cancer 2019;144:848-58.

34 Chang $\mathrm{H}$, Sasson A, Srinivasan S, et al. Bioinformatic methods and bridging of assay results for reliable tumor mutational burden assessment in non-small-cell lung cancer. Mol Diagn Ther 2019;23:507-20.

35 Kazdal D, Endris V, Allgäuer M, et al. Spatial and temporal heterogeneity of panel-based tumor mutational burden in pulmonary adenocarcinoma: separating biology from technical artifacts. $J$ Thorac Oncol 2019;14:1935-47.

36 Ricciuti B, Kravets S, Dahlberg SE, et al. Use of targeted next generation sequencing to characterize tumor mutational burden and efficacy of immune checkpoint inhibition in small cell lung cancer. $J$ Immunother Cancer 2019;7:87. 